\title{
What is statins legacy in chronic kidney disease patients?
}

\begin{abstract}
Although there is evidence that dyslipidemia plays a role, the intervention with statins, through their anti-lipid or pleiotropic effects, is still not clearly recommended to delay the progression of the renal disease.

Our aim was to analyze the eventual effect of statins in kidney disease progression. An observational retrospective study, 349 patients (mean age $=74.6$ years, females $=54.7 \%$ ) followed in a "low clearance" clinic with a baseline eGFR of $19.9 \mathrm{~mL} / \mathrm{min}$. Population was divided in two groups: $\mathrm{G} 1=$ under statins $(\mathrm{n}=158)$ and $\mathrm{G} 2=$ without statins $(\mathrm{n}=191)$. Kidney survival, defined by the time until commencement of dialysis, was compared between groups. Regarding statistical analysis, Student's t-test, $\chi^{2}$ test, Kaplan-Meier test and finally a logistic regression. G1 presented higher serum levels of calcium $(9.34$ vs $9.11 \mathrm{mg} / \mathrm{dL}, \mathrm{p}=0.004)$, albumin (4.04 vs $3.90 \mathrm{mg} / \mathrm{dL}, \mathrm{p}=0.018)$ and eGFR (21.57 vs $18.53 \mathrm{~mL} / \mathrm{min}, \mathrm{p}=0.004)$ and lower values of phosphorus $(4.00 v s 4.17 \mathrm{mg} / \mathrm{dL}, \mathrm{p}=0.044)$, PTH (206.08 vs $280.68 \mu \mathrm{g} / \mathrm{d}, \mathrm{p}=0.0001)$ and creatinine $(2.88 v s 3.52 \mathrm{mg} / \mathrm{dL}, \mathrm{p}=0.0001)$. The mean monthly dosis of vitamin $\mathrm{D}$ analogues $(2.10 \mathrm{vs} 6.87 \mu \mathrm{g}, \mathrm{p}=0.0001)$ and darbepoetin

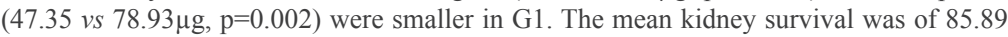
months in $\mathrm{G} 1$ and 65.88 months in $\mathrm{G} 2$ (Log Rank $=6.639, \mathrm{p}=0.010)$. The hemoglobin $(\mathrm{OR}=0.741$, CI 95\% 0.550-0.998, $\mathrm{p}=0.048)$, phosphorus $(\mathrm{OR}=1.988$, CI 95\% 1.205-3.280, $\mathrm{p}=0.007)$ and creatinine $(\mathrm{OR}=2.089$, CI 95\%1.162-3.754, $\mathrm{p}=0.014)$ were main predictors of kidney disease progression while the opposite occurred with statins' use (OR=0.372, CI $95 \% 0.198-0.701, p=0.002$ ). In our study, the use of statins demonstrated a potential benefit in reducing CKD progression. Hyperphosphatemia and lower levels of hemoglobin were independent predictors of progression to terminal CKD
\end{abstract}

Volume 9 Issue 4 - 2019

\author{
Filipa Brito Mendes,' Eduarda Carias,' André \\ Fragoso,' Ana Paula Silva, ${ }^{1,2}$ Pedro Leão \\ Neves ${ }^{1,2}$ \\ 'Nephrology Department, Centro Hospitalar e Universitário do \\ Algarve, Portugal \\ ${ }^{2}$ Department of Biomedical Science, Algarve University, Portugal
}

Correspondence: Filipa Brito Mendes, Centro hospitalar e universitário do algarve, Nephrology department, Portugal,Tel 0035 | 28989 | 100,Email: fbmendes@chalgarve.min-saude.pt, filiabriomendes@gmail.com

Received: July 23, 2019 | Published: August 06, 2019

Keywords: lipids, kidney disease, mineral metabolism

\section{Introduction}

Dyslipidemia has a high prevalence among patients with chronic kidney disease (CKD) and contribute to the elevated cardiovascular morbidity and mortality, as well as to the progression of the renal disease. Regarding statins renal protective role, owing to their lipid lowering effect, and also to their pleiotropic properties there is some controversy in the literature. ${ }^{1}$ The use of statins in earlier kidney disease stages could increase renal protection but it might be dosedependent. Some studies suggest that a more prolonged treatment could increase renal protection. ${ }^{1,2}$

The aim of this study was to assess the benefit of statins in patients with chronic kidney disease and evaluate the risk factors of progression of the renal disease until the initiation of dialysis.

\section{Patients and methods}

An observational retrospective study involving chronic kidney disease patients with a glomerular filtration rate of $<30 \mathrm{ml} / \mathrm{min} / 1.73 \mathrm{~m}^{2}$ conducted in the Low Clearance clinic at nephrology department of Centro Hospitalar e Universitário do Algarve-Faro since 2008 until 2012. Patients were divided in two groups according to statin therapy: Group1 (G1) under statins and Group 2 (G2) without statin therapy. Kidney survival, defined by the time until commencement of dialysis, was compared between groups.

The exclusion criteria used were an attendance at the consultation lower than three months and a recent history of neoplasia. Several laboratory parameters were analyzed: parathyroid hormone (PTH), calcium, phosphorus, cholesterol, HDL, triglycerides (TG), creatinine, albumin, hemoglobin and estimated GFR (MDRD formula). The serum levels of cholesterol, triglycerides, HDL, phosphorus and calcium were measured using ARCHITECT Systems and AEROSET System (Abbott Diagnostics Division, Abbott Laboratories Abbott Park, IL). Hemoglobin and PTH were calculated using spectrophotometry technique and Electro-chemiluminescence immunoassay (ECLIA) respectively. Monthly vitamin D intake, phosphorus binders and Cinacalcet were obtained through an hospitalar management system (Gestão Hospitalar de Armazém e Farmácia - GHAF) analyzes. The study was submitted to and approved by the administration and ethics committee. The study was conducted according to the principles of the Declaration of Helsinki, and study procedures were only performed after signing the informed consent.

\section{Statistics analyses}

We used descriptive statistics and for comparison between groups. We used student $t$ test and the $\chi^{2}$ test for continuous and categorical variables, respectively. The confirmation of a normal and homogenous distribution was by Kolmogorow-Smirnov and the Levene tests. The Kaplan-Meier method was used to measure the renal survival and for comparison between groups we used the log rank test. A multivariate logistic regression was used to determine the risk factors of kidney disease progression until the initiation of dialysis. Differences were considered statistically significant for $\mathrm{p}$-values $<0.05$. Statistical analysis was performed using SPSS 22 version (SPSS Inc., Chicago, IL, USA). 


\section{Results}

A total of 349 patients, being 191 female gender (54.7\%), all of them attending the Low Clearance clinic between 2008 and 2012. The mean of patient's age is 74.65 years. The estimated glomerular filtration rate was $19.90 \mathrm{~mL} / \mathrm{min}$ at baseline. Etiology of renal disease was unknown in $49.5 \%$; diabetes was the cause of renal disease in 29.8 $\%$, hypertension in $13.8 \%$, autosomal polycystic kidney disease in 2.3 $\%$ and glomerular diseases in $2.0 \%$. Table 1 presents the baseline population characteristics and the baseline laboratorial parameters. It can be observed that our population is quite old, and most of them $(97.1 \%)$ were under antihypertensive medication.

In Table 2 we compared the 2 groups regarding biological and laboratorial parameters. G 1 patients (under statin therapy), at baseline, had better renal function $(\mathrm{p}=0.004)$, as well as higher levels of calcium $(\mathrm{p}=0.004)$ and albumin $(\mathrm{p}=0.018)$ and lower levels of phosphate $(\mathrm{p}=0.044)$ and PTH $(0.0001)$. There were no differences regarding age, sex distribution, haemoglobin and the lipid profile. Expectedly G2 patients were treated with higher mean dose of vitamin $\mathrm{D}(\mathrm{p}=0.0001)$ and darbepoetin $(\mathrm{p}=0.0002)$. Using the Kaplan-Meier analysis, it was observed that the mean kidney survival in G1 was 85.89 months and in G2 was 65.88 months, with a $\log$ rank $=6.639$; $\mathrm{p}=0.010$ (Figure1). Using a logistic regression model, with dialysis initiation as the dependent variable, we found that haemoglobin $(\mathrm{OR}=0.741, \mathrm{p}=0.048)$, phosphorus $(\mathrm{OR}=1.988, \mathrm{p}=0.007)$ and creatinine $(\mathrm{OR}=2.089, \mathrm{p}=0.014)$ were found to be independent risk factors of dialysis initiation while the opposite occurred with the use of statins (OR=0.372, CI 95\% 0.198 to $0.701, \mathrm{p}=0.002)$ (Table 3).

Table I Demographic and laboratorial characteristics of the population

\begin{tabular}{ll}
\hline Characteristics & Values \\
\hline Number of patients included, $\mathrm{n}$ & 349 \\
Age, years & $74.65 \pm 13.92$ \\
Gender, M/F (\%) & $158 / 191(45.3 / 54.7)$ \\
Arterial systolic pressure $(\mathrm{mmHg})$ & $122.40 \pm 43.49$ \\
Arterial diastolic pressure $(\mathrm{mmHg})$ & $65.20 \pm 22.54$ \\
Hg (g/dL) & $11.49 \pm 1.19$ \\
Creatinine $(\mathrm{mg} / \mathrm{dL})$ & $3.24 \pm 1.29$ \\
GFR (ml/min) & $19.90 \pm 9.86$ \\
Cholesterol $(\mathrm{mg} / \mathrm{dL})$ & $160.07 \pm 64.62$ \\
Triglycerides $(\mathrm{mg} / \mathrm{dL})$ & $134.97 \pm 99.90$ \\
HDL (mg/dL) & $38.29 \pm 17.81$ \\
Phosphorus (mg/dL) & $4.10 \pm 0.78$ \\
Calcium (mg/dL) & $9.21 \pm 0.77$ \\
PTH (Pg/mL) & $256.05 \pm 201.50$ \\
Albumin $(\mathrm{g} / \mathrm{dL})$ & $4.00 \pm 0.48$ \\
Antihypertensive therapeutic S/N \% & $340 / 9(97.1 / 2.9)$ \\
\hline & \\
\hline
\end{tabular}

Table 2 Comparison of variables between GI and G2

\begin{tabular}{|c|c|c|c|}
\hline & $\begin{array}{l}\text { GI (under } \\
\text { statins) } n=\mid 58\end{array}$ & $\begin{array}{l}\text { G2 (without } \\
\text { statins) } n=\mid 91\end{array}$ & P value \\
\hline Age, Years & $74.24 \pm 13.82$ & $74.99 \pm 14.15$ & 0.14 \\
\hline Gender (f/m) & $79 / 79$ & $111 / 80$ & 0.133 \\
\hline $\mathrm{Hg}(\mathrm{g} / \mathrm{dL})$ & $11.6 \pm 1.16$ & II. $4 \pm 1.20$ & 0.14 \\
\hline Calcium (mg/dL) & $9.34 \pm 0.57$ & $9.11 \pm 0.89$ & 0.004 \\
\hline Phosphorus (mg/dL) & $4.00 \pm 0.63$ & $4.17 \pm 0.87$ & 0.044 \\
\hline PTH (pg/dL) & $206.08 \pm 159.59$ & $280.68 \pm 210.89$ & 0.0001 \\
\hline Creatinine (mg/dL) & $2.88 \pm 1.06$ & $3.52 \pm 1.40$ & 0.0001 \\
\hline GFR (ml/min) & $21.57 \pm 8.02$ & $18.53 \pm 10.97$ & 0.004 \\
\hline Cholesterol (mg/dL) & $|70,4| \pm 45.60$ & $179.18 \pm 56.82$ & 0.12 \\
\hline $\mathrm{HDL}(\mathrm{mg} / \mathrm{dL})$ & $52.39 \pm 25.27$ & $52.20 \pm 29.40$ & 0.95 \\
\hline TG (mg/dL) & $133.16 \pm 62.35$ & $|44.16 \pm 99.6|$ & 0.23 \\
\hline Albumin (mg/dL) & $4.04 \pm 0.35$ & $3.90 \pm 0.62$ & 0.018 \\
\hline AP Systolic (mm/Hg) & $125.30 \pm 38.68$ & $126.38 \pm 38.79$ & 0.79 \\
\hline AP Diastolic $(\mathrm{mm} / \mathrm{Hg})$ & $66.91 \pm 21.45$ & $67.60 \pm 20.94$ & 0.76 \\
\hline $\begin{array}{l}\text { Vitamin D monthly } \\
\text { intake }(\mu \mathrm{g})\end{array}$ & $2.10 \pm 6.78$ & $6.87 \pm 10.80$ & 0.0001 \\
\hline $\begin{array}{l}\text { Darbepoetin monthly } \\
\text { dosis }(\mu \mathrm{g})\end{array}$ & $47.35 \pm 80.22$ & $78.93 \pm 103.91$ & 0.002 \\
\hline
\end{tabular}

Table 3 Risk factors of dialysis initiation

\begin{tabular}{llll}
\hline & Adjusted OR $(95 \% \mathrm{Cl})$ & & P value \\
Statin use & $0.372(0.198-0.70 \mathrm{I})$ & & 0.002 \\
Age & $\mathrm{I} .006(0.98 \mathrm{I}-\mathrm{I} .03 \mathrm{I})$ & & $0.65 \mathrm{I}$ \\
Gender & $\mathrm{I} .076(0.5 \mathrm{I} 4-2.253)$ & & 0.846 \\
$\mathrm{Hg}$ & $0.74 \mathrm{I}(0.550-0.998)$ & & 0.048 \\
Phosphorus & $\mathrm{I} .988(\mathrm{I} .205-3.280)$ & & 0.007 \\
PTH & $\mathrm{I} .000(0.999-\mathrm{I} .002)$ & & 0.759 \\
Creatinine & $2.089(\mathrm{I} .162-3.754)$ & 0.014 \\
Albumin & $\mathrm{I} .049(0.559-\mathrm{I} .968)$ & $0.88 \mathrm{I}$ \\
\hline
\end{tabular}

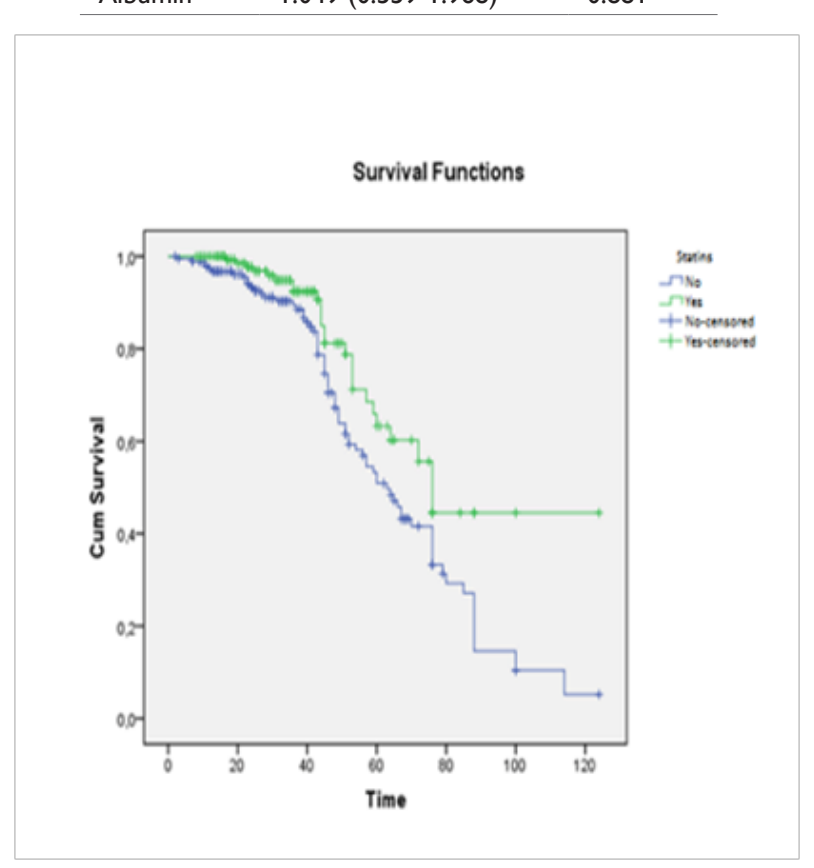

Figure I Renal survival. 


\section{Discussion}

Atherosclerotic cardiovascular disease is the main cause of morbidity and mortality in CKD patients. ${ }^{2,3}$ There is a bulk of evidence showing that, in the general population, dyslipidemia is associated with increased hard cardiovascular end-points, ${ }^{4}$ as well as with a greater prevalence of chronic kidney disease. ${ }^{5,6}$ Consequently, it is not controversial the use of statins in the general population with dyslipidemia. Nevertheless, the benefits of statins in patients with renal insufficiency are more elusive. ${ }^{?}$

As could be expected, HMG-CoA reductase inhibitors, also known as statins, have largely been investigated in order to clarify the eventual benefit of it according the different stages of CKD. Lately, in 2013, KDIGO- Clinical Practice Guideline for Lipid Management in Chronic Kidney Disease recommended prescription of statin in nondialysis-dependent CKD patients aged 50 years and over. In younger patients they recommended statin therapy whenever present an additional cardiovascular risk factors (listed in guidelines). Moreover, there is no evidence of benefit in patients with dialysis-dependent CKD which means those patients should not be initiated on statin. Although, if previously medicated, they should continue., ${ }^{8,9}$

Messow et al., ${ }^{10}$ developed a meta-analysis where the use of statins was evaluated attending three outcomes: major cardiovascular events, cardiovascular death and all-cause mortality. They consider statins are indicated in CKD3, probably indicated in CKD4, not indicated in CKD5/5D and probably indicated in patients with a functioning transplant. Messow et al., ${ }^{10}$ raised some possible explanations why statins become less effective preventing those outcomes as renal function declines. One is the pathophysiologic "shift" which CKD patients suffer from simply atherosclerotic to calcific arteriopathy and myocardial fibrosis throughout the disease. Other explanations based on the causes of death which are essentially heart failure and arrhythmias more than myocardial infarctions; the uremic related risk factors and lower baseline LDL present in more advanced renal disease which is not expected that statins do much.

Furthermore, in many prospective cohort studies, randomizedcontrol trials and meta-analyses there is evidence of statins' pleotropic effects, ${ }^{11,12}$ including their benefit in delaying CKD progression ${ }^{13}$ and reducing proteinuria. ${ }^{7}$ Some authors defend there are many specific renal effects of statins such as inhibition of mesangial cell proliferation; podocyte-protection; stem cell-activating and antifibrotic effects beyond the anti-inflammatory, haemodynamic and immunomodulatory effects involved in the kidney. ${ }^{14}$

Statins have been reported as ameliorate the inflammation, the oxidative stress and many investigations have been done such as the ability to slow down the rate of renal function decline, although the results have been controverse. ${ }^{15}$ Zhang et a., $1^{16}$ demonstrated that statins reduced proteinuria or all- cause mortality of non-end stage CKD and explains it making references to the protection of the vascular endothelium, stabilization of pre-existing atherosclerotic plaques, and the suppression of vascular inflammatory substances. ${ }^{16,17}$ Also a meta-analysis by Douglas et al., ${ }^{18}$ showed that statins reduced albuminuria and proteinuria in 13 of the 15 studies.

This study included only CKD patients in advanced stages of the disease (stages 4 and 5) and intended to study renal outcome. Nonetheless some limitations arise as it is an observational nonrandomized study. Different kind of statins were used (pravastatin, sinvastatin and atorvastatin) and we know that statins have different lipophilicity making some of them hydrophilic and others lipophilic and consequently conferring the ability to penetrate in different tissues.$^{19}$ For instances proteinuria reducing properties of atorvastatin (PLANET I e PLANET II). ${ }^{20}$ More than that, some authors suggest that statins may have a dose-related effect. ${ }^{12}$ Baseline characteristics were different, namely the patients under statins treatment had an higher glomerular filtration rate, but inclusion criteria was straightforward: $\mathrm{GFR}<30 \mathrm{ml} / \mathrm{min} / 1.73 \mathrm{~m}^{2}$. One must wonder if it was a coincidence or if statins therapy caused this disparity, delaying kidney disease progression. Koya et al., ${ }^{21}$ demonstrated that the use of statins protects kidney and prevents or delays the renal function loss. A renal mortality analysis evidenced that patients under statins have a higher survival and a logistic regression model determined not taking statins as predictive risk factor to the begin of dialysis.

In our study, the patients under statins therapy presented lower phosphorus and PTH serum levels. Hyperphosphatemia, low calcitriol levels and high PTH levels are recognized to aggravate renal disease progression and increase cardiovascular risk in CKD patients. ${ }^{22,23}$ As statins have a beneficial effect in mineral metabolism, the association between cardiovascular risk and the renal disease progression is alleged related to the mechanism modulation. ${ }^{24}$ The group of patients prescribed statins therapy had higher albumin serum levels. Despite being a nutritional status index, related to protein intake, its higher levels can also correlate with lower urinary losses, as it is an attributable beneficial effect of statins. ${ }^{7,20}$ Statins seem to have a synergic effect with erythropoiesis stimulating agents (ESA) lowering their consumption, in accordance to the Koc M et al., ${ }^{25}$ study result. In fact in our population, there were no differences in hemoglobin levels, but it was due to higher dosages of ESA in the group without statins prescription.

This work shows a higher renal survival in patients on statins. These patients had higher baseline kidney function, calcium and albumin levels, and lower phosphorus and PTH levels. Logistic regression model shows that lower hemoglobin serum levels and higher levels of phosphorus and creatinine were predictive risk factors for the renal disease progression, but also that lack of statins therapy is associated with earlier dialysis initiation. Furthermore, prescription of statin therapy did not seem to ameliorate lipid profile, as there were no differences in lipid levels between groups, so one must think, that all of these beneficial effects on kidney disease must arise from pleotropic characteristics, modulation mechanisms or synergic effects.

\section{Conclusion}

In conclusion, our results show that the use of statins plays a role in renoprotection through an increase in renal survival. Its absence was predictor of earlier dialysis in a population with chronic kidney disease in stage 4 and 5. Statins also improved bone mineral metabolism and albumin levels. We believe that a larger sample size and the inclusion of more variables in the study could provide more accurate results, allowing the achievement of more important data. Despite the relatively small sample and the limited power of the analyzes, further studies can be designed based on the results shown in order to clarify the role of statins and type of statin on the progression of renal disease as well as their pleiotropic effects on secondary complications.

\section{Acknowledgment}

All authors of this research paper have directly participated in the planning, execution or analysis of this study. 


\section{Conflicts of interest}

The author declares there are no conflicts of interest.

\section{Funding details}

None.

\section{References}

1. Nikolic D, Banach M, Nikfar S, et al. A meta-analysis of the role of statins on renal outcomes in patients with chronic kidney disease. Is the duration of therapy important? Int J Cardiol. 2013;168(6):5437-5447.

2. Fan D, McCulloch CE, Hsu CY. Chronic kidney disease and the risks of death, cardiovascular events and hospitalization. $N$ Engl J Med. 2004;351:1296-1305.

3. Collins AJ, Foley RN, Herzog C, et al. US Renal Data System 2010 Annual Data Report. Am J Kidney Dis. 2011;57(suppl 1):e87-e98.

4. Castelli WP, Anderson K, Wilson PW, et al. Lipids and risk of coronary heart disease. The Framingham Study. Ann Epidemiol. 1992;2:23-28.

5. Manttari M, Tiula E, Alikoski T, et al. Effects of hypertension and dyslipidemia on the decline of renal function. Hypertension. 1995;26:670-675.

6. Muntner P, Coresh J, Smith JC, et al. Plasma lipids and risk of developing renal dysfunction: The Atherosclerosis Risk in Communities Study. Kidney Int. 2000;58(1):293-301.

7. Guedes AM, Neves PL. Statins for renal patients: a fiddler on the roof? Int J Nephrol. 2012;2012:806872.

8. Gerald B Appe. Lipid abnormalities in patients with chronic kidney disease not requiring dialysis. Uptodate. 2013.

9. KDIGO Clinical Practice Guideline for Lipid Management in Chronic Kidney Disease. 2013.

10. Messow CM, Isles C. Meta-analysis of statins in chronic kidney disease/ who benefits? QJM: An International Journal of Medicine. 2017.

11. Davignon J. Pleiotropic effects of pitavastatin. Br J Clin Pharmacol. 2012;73(4):518-535.

12. Kostapanos MS, Liberopoulos EN, Elisaf MS. Statin pleiotropy against renal injury. J Cardiometab Syndr. 2009;4(1):E4-E9.
13. Hou W, Lv J, Perkovic V, et al. Effect of statin therapy on cardiovascular and renal outcomes in patients with chronic kidney disease: a systematic review and meta-analysis. Eur Heart J. 2013;34(24):1807-1817.

14. Amann K, Benz K. Statins-beyond lipids in CKD. Nephrol Dial Transplant. 2011;26:407-410.

15. Inoue $\mathrm{T}$, Ikeda $\mathrm{H}$, Nakamura $\mathrm{T}$, et al. Potential benefit of statin therapy for dyslipidemia with chronic kidney disease: Fluvastatin Renal Evaluation Trial (FRET). Intern Med. 2011;50(12):1273-1278.

16. Sharp Collaborative Group. Study of Heart and Renal Protection (SHARP): randomized trial to assess the effects of lowering low-density lipoprotein cholesterol among 9,438 patients with chronic kidney disease. Am Heart J. 2010;160(5):785-794.

17. Honore PM. Statins and the Kidney: Friend or Foe? Blood Purif. 2017;43:91-96.

18. Guedes AM, Neves PL. Statins for renal patients: a fiddler on the roof? Int J Nephrol. 2012;2012:806872.

19. Upadhyay A, Earley A, Lamont JL, et al. Lipid-lowering therapy in persons with chronic kidney disease: a systematic review and metaanalysis. Ann Intern Med. 2012;157(4):251-262.

20. Kalaitzidis RG, Elisaf MS. The Role of Statins in Chronic Kidney Disease. Am J Nephrol. 2011;34:195-202.

21. Koya D, Campese VM. Statin use in patients with diabetes and kidney disease: the Japanese experience. J Atheroscler Thromb. 2013;20(5):407424.

22. Martin KJ, Gonzalez EA. Prevention and control of phosphate retention/ hyperphosphatemia in CKD-MBD: what is normal, when to start, and how to treat? Clin J Am Soc Nephrol. 2011;6(2):440-446.

23. Alfred K Cheung, William L Henrich. Secondary prevention of cardiovascular disease in end-stage renal disease (dialysis). Uptodate. 2013.

24. Stompor T, Olszewski A, Kierzkowska I. Can we prolong life of patients with advanced chronic kidney disease: what is the clinical evidence? Pol Arch Med Wewn.2011;121(3):88-93.

25. Koc M, Dogan C, Arinsoy T, et al. Statin use is associated with lower inflammation and erythropoietin responsiveness index in hemodialysis patients. Hemodial Int. 2011;15(3):366-373. 